\title{
Sedimentary micro-phase and reservoir display characteristics in shale Oil
}

\author{
Cai Xiao ${ }^{1,2}$ \\ ${ }^{1}$ The Key Laboratory of Unconventional Oil \& Gas geology, CGS; China \\ ${ }^{2}$ SINOPEC East China Oil and Gas Company Exploration and Development Research Institute China
}

\begin{abstract}
Sedimentary microphilic and its exhibition feature is of great significance for identifying the distribution of remaining oil. This paper studies the development of geological characteristics of Zhidan Oil Zone, mineralogue and scanning electron microscopy experiments, and obtains the sedimentary micro-phase and reservoir display characteristics. The results show that combined with logging data, the core phase analysis, logging phase analysis, single well phase analysis, etc. Taking the leading edge of the Delta, the microphase type such as the river, the diversion bay and the underwater natural embankment is developed under water, due to the lifting of the lake, form a number of invested, plus, replenishment, in which water The downstream river is facilitating the advantages of the reservoir. The sedimentary phase and the angle effect of the zone are dominated by $4+5$, chang 6 oil and gas, showing the characteristics of the rock sex reservoir. The type of reservoir is a rock sex reservoir. The main development of sandstone is impaired and the sandstone lens is a rock sex reservoir. The reservoir is an important influencing factor in the reservoir. The area has no uniform oil and water interface, lacking edge, bottom water.
\end{abstract}

Keywords: Zhidan oil area; extended group; deposition; reservoir exhibition.

\section{Introduction}

Understanding the sedimentary environment, deposition microforming and exhibition feature of the middle and later reservoirs, which have important practical significance for identifying the distribution of remaining oils, and the post-injection well network design is important. Through the system collection and organizing the core, recording of the study area And a large number of logging materials, the formation is detailed, and there is an important research significance to further clarify the feature of the reservoir. Zhidan oil plant 3043 well area is located in the northeastern part of Zhidan County, with an area of about $33 \mathrm{~km} 2$ and 723 in total wells. Among them, there is 166 injection wells, 557 oil mining well; current average single well Nissan liquid $0.84 \mathrm{~m} 3$, Nissan oil 0.37 T, $48 \%$ water; in which the water moisture is $\geq 80 \%$ oil well 40 , due to water flooding, high water stops lying in the well 70; the main oil layer is $4+5$ and chang 6 oil layer group, and some areas simultaneously develop length 3 and lower Combined oil layer group. This paper studies the development of geological characteristics of Zhidan Oil Zone, mineralogue and scanning electron microscopy experiments, and obtains the sedimentary micro-phase and reservoir display characteristics.

\section{Method and experiment}

\subsection{Sedimentary microphase division}

Combined with the recording phase, the logo phase and the seismic phase analysis are the main methods and means of the current sedimentary microformation analysis. Conventional deposition microphase analysis mainly includes a combination analysis of rock sex, core deposition characteristics, heavy mineral vertical and horizontal planar display characteristics, ancient water flow analysis, etc. At present, the system binds to the sedimentary microphase analysis technology, and the deposition microformation method of the logo phase, the log phase and the seismic phase has been widely used. From the perspective of longitudinal research accuracy, it is carried out in a sand group or Sedimentary microplasma analysis of single sand layer is the largest formation unit of oil and gas release exploration. From a specific deposition microphase planar view technique, for the same deposition microformation study formation unit (such as sand group or single sand layer), a well point conventional deposition microformation, recording phase and logging phase analysis The starting point determines the deposition microphase type, and the study results of the study of sandstone thickness, the planar extrapolation 
is determined to determine the deposition micro phase boundary on the plane. When planned, the sedimentary micropomposition is selected as the oil and gas reservoir relationship is a superior phase, indicating a percentage of different deposition microspices in longitudinal direction. The deposition microphase type facilitating the development of the oil and gas is then determined by the deposition microphase type analysis belonging to the oil reservoir to be known. On the basis of the former research work, the core of the center of the work area was observed and the core phase analysis was formed, forming a comprehensive understanding of the deposition of $4+5$, chang 6 oil layers in the 359 well region. Its research methods are as follows (Figure 1):

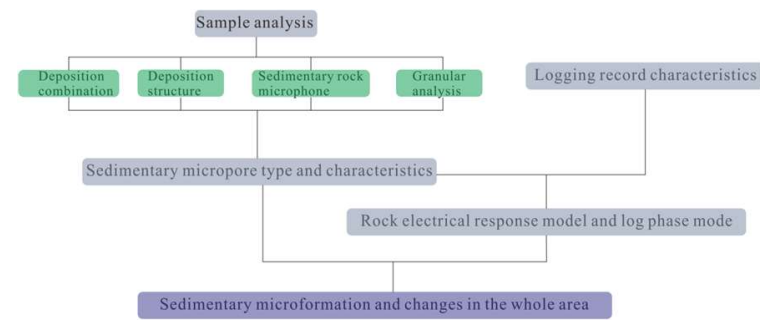

Figure 1 Sedimentary microphlic research flow chart

\subsection{Scanning Electron Microscopy (SEM)}

The scanning electron microscope experiment was carried out at China University of Petroleum (Beijing). The model used was FEI Quanta 200. The sample was observed by argon ion polishing before the observation. In the experiment, the energy type of the shale was combined with the energy spectrometer to determine the pore type in the shale. The resolution is $2 \mathrm{~nm}$.

\section{Results and discussion}

\subsection{Rock phase sign and rocky characteristics}

The rock phase is the sum of the rocky characteristics formed in a particular deposition environment, the same rock represents the product under the same deposition conditions. By analyzing the characteristics of different rocks, structures, deposition structures, types, etc., can reflect the hydraulic conditions of different abrasive depositions, and restore the original deposition environment. According to the core observation, the zone develops 8 major rock types.

(1) Block layer: mainly gray sandstone (Figure 2), single layer thickness is small, bottom is a mutation contact, the layers are not obvious, the up transition is the parallel layer, and it is slightly thinner. The rhyme rhythm is rotated back, reflecting the rapid accumulation under strong water conditions, mainly in the river in the front of the Delta.

(2) Parallel layer: mainly gray fine sandstone (Figure 3, Figure 4), a single layer thickness, better sorting. The thickness of the tattoo layer is between $0.2 \sim 0.5 \mathrm{~cm}$, which consists of parallel flat continuous or intermittent texture, and the texture is displayed by char. Reflects the water-motion conditions of water flow. It is mainly seen in the leading water under the front of the Delta. .
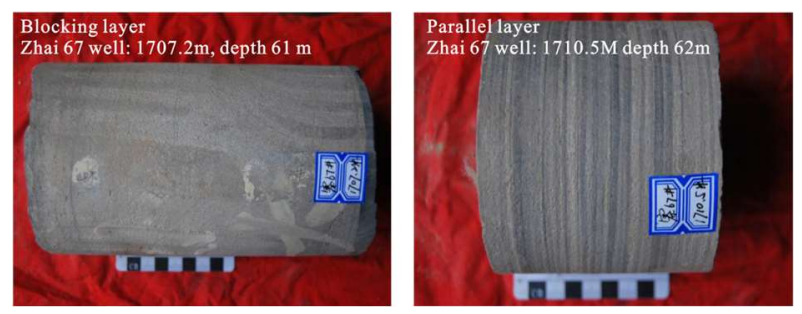

Figure 2 Block layerFigure 3 Parallel layer

(3) Interlaced layers: Mainly gray fine sandstone (Figure 5), a single layer thickness, better sorting. Composed of a series of slanting layers, the texture is displayed by charging, reflects strong hydraulic conditions, mainly seen in the front of the Delta frontal water.

(4) Horizontal layers: Mainly developed in mudstone, mudstone, muddy powder sandstone. Its texture is thin and parallel to each other, indicating that in low flow of low energy, forms a suspension substance deposition, and is common in diversion bay deposition.

(5) Deformation Hierarchical: Common and Sandstone and fine sandstone, belonging to the layers of layers in the layer. Due to the liquefaction effect in the deposited layer or the shear effect of the water, it is common in the deposition of the shunt.

(6) Sand pattern layer: there is a sand-shaped migration. Mainly in the sandstone, muddy powder sandstone, is a multi-layered small small interleaving layer, and the lower interface of the layer is micropro-waveform, the tattoo is irregular, intermittent or continuous, and the fine layer is inclined to one side and Under the convergence, see fine plant debris, carbonia and rich mica tablets, and often with parallel layers, plate-shaped staggery and small interlaced layers. It is mainly formed in an environment with weak hydrodynamic conditions, such as the Delta Division Diversion Bay Deposition Environment.

(7) Cycloma: Commonly in the sand mud, multiple development in powder $\sim$ fine sandstone, muddy powder sandstone and sand mudstone, fine layer is curved, sometimes clearly lenticular, corrugated The mud layer and the sand layer are unregulated to each other in front of the Delta premium sand deposition.

(8) Flush surface structure: It is a deposition structure that is common in the study area. It is a surface of strong water to form unevenness in the surface of the dodel. It is shown in the mudstone, and the sandstone is stuck in sandstone. And more mud and tear muddy fragments. The flushing surface is generally developed at the bottom of the river. When there are multiple discarded rivers to overlap each other, there can be seen a plurality of mudble layers.

According to the observation and statistics of the sheet and the surface electron microscope, the length of the Zhidan Oilfield 3043 well regions extended $4+5$ and chang 6 reservoir rock types are mainly light gray, gray, gray brown, gray green for powder - fine granules and Medium - fine grain chute. The ingredients are main, the stone is the stone, the dark mineral is small, the selection is good, the mud gel, the subprogramme, and the 
looseness. The overall mineral maturity is low, the structure is high, and the rocky characteristics of the formation of a strong sandstone reservoir.

\subsection{Sedimentary type}

According to the above deposition phase identification method, the predecessor is taken from the large number of geological studies in the region, and the sedimentary microforming of the length of $4+5$ and 6 in the region is divided. The length $4+51$ in the area is the Delta Plain Asia, and the natural embankment, the natural embankment, the river is deposited; the length of $4+52$, chang 6 , the period is the leader of the Delta, subdivided into the water Divide the river, underwater natural embankment, the split bay deposited microphones. According to regional data, core observation and logging data analysis can be divided into the leading edge deposition system in the Delta, and the detailed division is shown in Table 1.

1. Underwater shunt

The research area underwater shunt river sand body is an important reservoir, and the water shifts in the water is more straightforward. The more it is more smaller. At the bottom of its deposition, there is often an unevenly scored surface, and there are more mud and mudstone tore. In the rock section, the river props are multi-rhythm. The single sand layer has a small thickness, which exhibits a tendency to gradually thinner from the main river to the river wing, and the uniform block shape of the positive rotary or top mutation is generally present. The obvious mud rock compartment, but the particle size distribution is unstable, mostly medium, fine, abrasive thin layer interactive body, and local muddy strip development. The natural potential curve is a bell or box shape, represents the deposition of the side, and the plus. The natural gamma curve curve is strong and abnormal, the microelectrode is large, and the permeability is good. The sound speed curve is low, dental, but has a peak. The resistivity curve is a medium amplitude wave.

Table 13043 Well Region chang 4 + 5, chang 6 Deposition System Division

\begin{tabular}{|c|c|c|c|c|}
\hline \multicolumn{2}{|c|}{ Layout } & phase & Subphor & Microphone \\
\hline \multirow[t]{2}{*}{ Chang $4+5$} & Chang $4+5^{1}$ & \multirow{6}{*}{ Delta } & $\begin{array}{l}\text { Delta } \\
\text { plain }\end{array}$ & $\begin{array}{l}\text { Distilted river, } \\
\text { natural } \\
\text { embankment, } \\
\text { river }\end{array}$ \\
\hline & Chang4+5 & & \multirow{5}{*}{$\begin{array}{l}\text { Delta } \\
\text { front } \\
\text { edge }\end{array}$} & \multirow{5}{*}{$\begin{array}{c}\text { Underwater shunt } \\
\text { Underwater } \\
\text { natural } \\
\text { embankment } \\
\text { Diversion Bay }\end{array}$} \\
\hline \multirow{4}{*}{ Chang6 } & Chang $6^{1}$ & & & \\
\hline & ${\text { Chang } 6^{2}}^{2}$ & & & \\
\hline & Chang6 $^{3}$ & & & \\
\hline & ${\text { Chang } 6^{4}}^{4}$ & & & \\
\hline
\end{tabular}

\section{2 , divide the river}

It is part of the grid in the Delta plain, with the characteristics of general river deposition, mainly laminated fine sandstone, laminated, laminated sandstone, regular rhythm. The sandstone sorting and molarity is good, and the debris particles are circular or rounded. Generally, the bottom is medium $\sim$ fine sand sand, often containing the sediments such as mud, plant stems, and the upwards of sanding, muddy powder sandstone and powder sand mud. The sanding surface is seen in the bottom of the sand, and the sand layer has a slot or plateshaped interlaced hierarchy and a wave interlaced layer. The cross section is a top bottom convex or double convex lens shape, and the sand body is often sinking in a downstream mudstone layer, wherein the outermost thickness and thickening are thinned to both ends. The natural potential curve is mostly a box-shaped negative abnormality, and there is a bell shape, a bell-shaped box and a box-shaped $\sim$ funnel is negative.

3 , natural embankment

On the land on the land. It is usually seen in the longitudinal section and is located on both sides of the shunt main river bed, and its morphology is controlled by the main river channel. The lithology is mostly a sandstone and muddy powder sandstone, and the thin layer is thin sandstone and mudstone. Cyber layer, sand grain interleaving layer, horizontal hierarchy and water wave marks are more common. The natural potential curve and the natural gamma curve are low in a lower center finger, which is a plurality of funnel or step-shaped stacks, and from the main river road to the river wing, the bell shape is gradually increased, the curve shape gradually became irregular zigzag, curved amplitude Small.

\subsection{Reservoir type}

The sedimentary phase and the angle effect of the zone are dominated by $4+5$, chang 6 oil and gas, showing the characteristics of the rock sex reservoir. The type of reservoir is a rock sex reservoir. The main development of sandstone is impaired and the sandstone lens is a rock sex reservoir. The reservoir is an important influencing factor in the reservoir. The area has no uniform oil and water interface, lacking edge, bottom water.

(1) Sandstone tilt rocky reservoir

Such reservoirs are widely distributed, in the horizontal length of $4+5$, the chang 6 oil layer group Delta frontal water is divided into the shunt of the river sand body in the upper direction of the bay mudstone, and the reservoir superficial direction sandstone is destroyed. Gravity is a dense powder sandstone, mudstone. The reservoir sandstone side flaps and the upper direction of the reservoir (mudstone) are formed on the plane.

(2) Sandstone lens body rock sex reservoir

This type of reservoir is more developed in this region, mainly with mud mud rock, sandstone, and sandstone body generally lenticular output, and the reservoir feature is coincident with sand body development, generally a lens-shaped distribution.

\section{Conclusion}

(1) On the basis of mound heart observation, combined with logging data, the core phase analysis, log finding analysis, single well phase analysis, etc., the logging phase recognition mode is established, and the 3043 well region length $4+$ is obtained. 5. chang 6 oil layer group mainly based on the leading edge of the Delta, and the 
microformation of the river, the diversion bay and the underwater natural embankment is developed. Due to the rise and fall of the lake, a number of invested, plus, The replenishment is rotated back, wherein the water split river is facilitating the advantages of the reservoir. The research section leads $4+5$ and chang 6 reservoir rock types are mainly long-stone sandstone, and the debris ingredients are mainly stone and quartz, followed by black microconuts and stings. The debris particles are mainly in line contact and point-line contact, and local visible points are in contact. The cementation type is mainly pore cement, thin film cement, film-porous cement, and increase-pore cement, a small amount of embedded cement. The pore type is mainly granulated hole and a variety of dissolves, a small amount of crack holes.

(2) The sedimentary phase and the angle effect of the region are dominated by $4+5$ and chang 6 oil and gas, showing the characteristics of the rock sex reservoir. The type of reservoir is a rock sex reservoir. The main development of sandstone is impaired and the sandstone lens is a rock sex reservoir. The reservoir is an important influencing factor in the reservoir. The area has no uniform oil and water interface, lacking edge, bottom water.

\section{Acknowledgments}

Thanks to the Funding support by Study on the microscopic pore structure and adsorption capacity of organic matter and clay minerals in shale ( Number: DD20160178-03-02).

\section{References}

1. Yang F, Ning Z F, Hu C P, et al. Characterization of microporous pore structures in shale reservoirs[J]. Acta Petrolei Sinica, 2013, 34(2): 301-311.

2. Bustin R M, Bustin A M M, Cui A, et al. Impact of shale properties on pore structure and storage characteristics $[\mathrm{C}] / / \mathrm{SPE}$ shale gas production conference. Society of Petroleum Engineers, 2008.

3. Jiao K., Yao S., Wu H., etc. Research progress on characterization methods of shale gas reservoir pore system [J]. Geological Journal of China Universities, 2014(01): 151-161.

4. Zhou S, Yan G, Xue H, et al. 2D and 3D nanopore characterization of gas shale in Longmaxi formation based on FIB-SEM[J]. Marine and Petroleum Geology, 2016, 73: 174-180.

5. Dung Le T, Murad M A. A new multiscale model for methane flow in shale gas reservoirs including adsorption in organic nanopores and on clay surfaces[C]//International Symposium on Energy Geotechnics (1st: 2015: Barcelona). Universitat Politècnica de Catalunya. Departament d'Enginyeria del Terreny, Cartogràfica i Geofísica, 2015.

6. Wei M, Zhang L, Xiong Y, et al. Nanopore structure characterization for organic-rich shale using the non- local-density functional theory by a combination of $\mathrm{N}$ 2 and $\mathrm{CO} 2$ adsorption[J]. Microporous and Mesoporous Materials, 2016, 227: 88-94.

7. Misch D, Mendez-Martin F, Hawranek G, et al. SEM and FIB-SEM investigations on potential gas shales in the Dniepr-Donets Basin (Ukraine): pore space evolution in organic matter during thermal maturation[C]//IOP Conference Series: Materials Science and Engineering. IOP Publishing, 2016, 109(1): 012010.

8. $\mathrm{Ju} \mathrm{B}, \mathrm{Wu}$ D. Experimental study on the pore characteristics of shale rocks in Zhanhua depression[J]. Journal of Petroleum Science and Engineering, 2016, 146: 121-128.

9. Yang, S, Wang, J, Liu, G, Li H. (2019). Horizontal well of shale gas complex fracturing casing failure mechanism., 944, 898-902.

10. Xiong J, Liu X, Liang L. Experimental study on the pore structure characteristics of the Upper Ordovician Wufeng Formation shale in the southwest portion of the Sichuan Basin, China[J]. Journal of Natural Gas Science and Engineering, 2015, 22: 530539.

11. Leng, Aolin, Liu, Zihan, Xing, Guangyuan, et al. China's Investment Incentive Strategy for Shale Gas Development[J]. Emerging Markets Finance and Trade, 2019(6):1-14.

12. Guo, Xue Li, Li, Jun, Zeng, Yi Jin, et al. Analytical Method to Evaluate Casing Stress during MultiFracturing for Shale Gas Wells[J]. 2019, 944:10501060.

13. Wang M, Yang J, Wang Z, et al. Nanometer-Scale Pore Characteristics of Lacustrine Shale, Songliao Basin, NE China[J]. PloS one, 2015, 10(8): e0135252.

14. Wan Y, Pan Z, Tang S, et al. An experimental investigation of diffusivity and porosity anisotropy of a Chinese gas shale[J]. Journal of Natural Gas Science and Engineering, 2015, 23: 70-79.

15. Curtis M E, Cardott B J, Sondergeld C H, et al. Development of organic porosity in the Woodford Shale with increasing thermal maturity[J]. International Journal of Coal Geology, 2012, 103: 2631.

16. Fishman N S, Hackley P C, Lowers H A, et al. The nature of porosity in organic-rich mudstones of the Upper Jurassic Kimmeridge Clay Formation, North Sea, offshore United Kingdom[J]. International Journal of Coal Geology, 2012, 103: 32-50.

17. Lowell S, Shields J E, Thomas M A, et al. Characterization of porous solids and powders: surface area, pore size and density[M]. Springer Science \& Business Media, 2012.

18. Clarkson C R, Solano N, Bustin R M, et al. Pore structure characterization of North American shale gas reservoirs using USANS/SANS, gas adsorption, and mercury intrusion[J]. Fuel, 2013, 103: 606-616. 